\title{
Clinical Characteristics and Outcomes of Patients with Acute Coronary Syndrome and Prior Coronary Artery Bypass Grafting in a Large Middle Eastern Cohort
}

\author{
Muath Alanbaei ${ }^{1, *}$, Alawi A. Alsheikh-Ali ${ }^{2}$, Tareq Aleinati ${ }^{3}$, Mohammad Zubaid ${ }^{1}$, Mustafa Ridha ${ }^{4}$,
} Fahad Alenezi ${ }^{4}$, Wael Al-Mahmeed ${ }^{5}$, Kadhim Sulaiman ${ }^{6}$, Jawad Al-Lawati ${ }^{7}$ Haitham Amin ${ }^{8}$, Jassim Al Suwaidi ${ }^{9}$ and Ahmed Al-Motarreb ${ }^{10}$

${ }^{1}$ Department of Medicine, Faculty of Medicine, Kuwait University, Kuwait

${ }^{2}$ Institute of Cardiac Sciences, Sheikh Khalifa Medical City, Abu Dhabi, United Arab Emirates and Tufts Clinical and Translational Science Institute, Tufts Medical Center, Boston, MA, USA

${ }^{3}$ Department of Cardiac Surgery, Chest Diseases Hospital, Ministry of Health, Kuwait

${ }^{4}$ Division of Cardiology, Department of Medicine, Adan Hospital, Ministry of Health, Kuwait

${ }^{5}$ Institute of Cardiac Sciences, Sheikh Khalifa Medical City, Abu Dhabi, United Arab Emirates

${ }^{6}$ Royal Hospital, Muscat, Oman

${ }^{7}$ Department of Non-communicable Diseases Control, Ministry of Health, Muscat, Oman

${ }^{8}$ Mohammed Bin Khalifa Cardiac Centre, Manama, Bahrain

${ }^{9}$ Hamad General Hospital and Hamad Medical Corporation, Doha, Qatar

${ }^{10}$ Department of Medicine, Faculty of Medicine, Sana'a University, Sana'a, Yemen

\begin{abstract}
Background: Acute Coronary Syndrome (ACS) can occur in patients with prior coronary artery bypass grafting (CABG). In the Gulf Registry of acute coronary events (Gulf RACE), we identified the clinical characteristics and inhospital outcomes of these patients.

Methods: Clinical characteristics and in-hospital outcomes for 461 ACS patients with prior CABG are compared to 7715 ACS patients without prior CABG enrolled from 64 hospitals in 6 Gulf countries over a 6-month period.

Results: The overall incidence of ACS with prior CABG was $5.6 \%$ out of 8176 patients. The ACS with prior CABG were older (63 vs 55 years, $P<0.0001$ ), had more history of diabetes $(62.3$ vs $37.6 \%, P<0.0001)$, dyslipidemia $(70.3$ vs $29.5 \%$, $P<0.0001)$, and hypertension $(75.7$ vs $47.8 \%, P<0.0001)$ compared with the non-CABG group. They presented more frequently with dyspnea (14.8 vs $9.5 \%, P<0.0005)$, non-ST segment elevation myocardial infarction $(41.4$ vs $31.6 \%$, $P<0.0001)$ and echocardiographic evidence of left ventricular dysfunction $(49.4$ vs $29.8 \%, P<0.0001)$ than ACS without prior CABG. They had a complicated in-hospital course with more recurrent ischemia (13.9 vs $9.3 \%, P=0.0011)$, heart failure ( 24.1 vs $15.7 \%$ ), and stroke ( 2.2 vs $0.6 \%$ ) compared with those without CABG. The in-hospital mortality rate was $5.6 \%$ in the $\mathrm{CABG}$ group compared with $3.5 \%$ in the ACS without prior CABG group. After adjusting for confounders, prior $\mathrm{CABG}$ was independently associated with recurrent ischemia and shock, more in patients presenting with ST elevation than non-ST elevation ACS.

Conclusions: Patients with ACS and prior CABG are a high-risk group with poor outcomes irrespective of their older age and comorbidities. They should be identified and treated differently to improve their outcomes.
\end{abstract}

Keywords: Acute Coronary Syndrome, Angioplasty, Comorbidity, Coronary Artery Bypass Grafting, Risk factors, Stroke.

\footnotetext{
*Address correspondence to this author at the Faculty of Medicine - Kuwai University, Medicine Department - Forth floor P.O. Box 24923 - Safat 13110 Kuwait; Tel: (965) 24986353; Fax: (965) 25318454;

E-mail: muath.alanbaei@hsc.edu.kw
}

\section{INTRODUCTION}

Increasing numbers of coronary artery bypass grafting (CABG) surgery was reported annually from the mid- to 
late-1990s in the United States and Europe [1]. Their benefit in reducing morbidity and mortality is documented [2, 3], with excellent short- and mid-term outcomes despite marked change in patient risk profiles and surgeons operating yearon-year on an increasing number of higher risk patients [4]. However, recurrent myocardial ischemia due to progression of atherosclerosis in the native coronaries or graft failure is common [5]. As a result the global burden of patients admitted with prior history of CABG and acute coronary syndrome (ACS) has increased in recent years [6].

A few high quality prospective controlled studies have examined the clinical impact of ACS in patients with prior CABG [6-8]. However, none of these studies was performed in Middle Eastern Arab countries. We have previously demonstrated that our ACS patients have different characteristics and receive less aggressive interventions compared to western ACS patients, with only a minority of ACS patients undergoing coronary angiography during the index hospitalization [9]. The goal of this study was to examine the characteristics, clinical presentations, treatment, and in-hospital outcomes of ACS with prior CABG in our region.

\section{MATERIALS AND METHODOLOGY}

\section{Study Population}

The Gulf Registry of acute coronary events (Gulf RACE) is a 6-month prospective, multinational study of all consecutive patients hospitalized with the final diagnosis of ACS in
64 centers in 6 Arab Gulf countries (Kuwait, Oman, United Arab Emirates, Yemen, Qatar and Bahrain). Patients were recruited in a pilot phase starting on 8 May 2006 for 30 days. This period was followed by 5 months recruitment starting on January 29, 2007. Full details of the methods have been previously published $[9,10]$. The term ACS included unstable angina, ST-segment elevation myocardial infarction (STEMI) and non-ST-segment elevation myocardial infarction (NSTEMI). For the purpose of this analysis, we examined patients with and without history of prior CABG surgery. The study received ethical approval from the institutional ethical bodies in all participating countries.

\section{Statistical Analysis}

Continuous variables are presented as median $\left[25^{\text {th }}-75^{\text {th }}\right.$ percentiles], and compared with the Wilcoxon rank-sum test. Categorical variables are presented as percentages, and compared with the Chi-square test. Multivariate logistic regression examined the association between prior CABG and inhospital outcomes adjusting for baseline characteristics that were significantly different at the univariate level between the 2 cohorts (with vs without prior CABG). To assess whether type of ACS (e.g. STEMI) modified the relationship between prior CABG and in-hospital outcomes, we tested for interaction with ACS type in the association between prior CABG and in-hospital outcomes. All associations from the logistic regression models are quantified as odds ratios (OR) with $95 \%$ confidence intervals (CI). SAS 9.1 statistical pack-

Table 1. Baseline Patient Characteristics and Comorbidities of the Study Cohort

\begin{tabular}{|c|c|c|c|}
\hline \multicolumn{4}{|l|}{ Demographics } \\
\hline Male \% & 76.0 & 71.4 & 0.03 \\
\hline \multicolumn{4}{|l|}{ Past Medical History (\%) } \\
\hline MI & 21.8 & 66.8 & $<0.0001$ \\
\hline PCI & 10.5 & 32.5 & $<0.0001$ \\
\hline \multicolumn{4}{|l|}{ Risk Factors (\%) } \\
\hline Current smoker (within a year) & 37.8 & 14.8 & $<0.0001$ \\
\hline Hypertension & 47.8 & 75.7 & $<0.0001$ \\
\hline Dyslipidemia & 29.5 & 70.3 & $<0.0001$ \\
\hline \multicolumn{4}{|l|}{ Comorbidities (\%) } \\
\hline COPD & 5.1 & 8.9 & 0.0012 \\
\hline Stroke & 4.3 & 9.5 & $<0.0001$ \\
\hline Dialysis & 0.9 & 2.0 & 0.047 \\
\hline PAD & 2.0 & 9.5 & $<0.0001$ \\
\hline
\end{tabular}

MI: myocardial infarction, PCI: percutanous coronary intervention, DM: diabetes mellitus, COPD: chronic obtructive pulmonary disease, PAD: periphral arterial disease. 
age (SAS Institute, Cary, NC, USA) was used for all analyses and a $\mathrm{P}<0.05$ was considered significant.

\section{RESULTS}

We enrolled 8,176 consecutive patients with ACS, of which 461 patients $(5.6 \%)$ had prior CABG. Patients with prior $\mathrm{CABG}$ had significantly more adverse baseline characteristics than those without CABG (Table 1). When compared with non-CABG ACS patients, ACS patients with prior $\mathrm{CABG}$ were older [63 vs 55 years, $\mathrm{p}<0.0001$ ], and significantly more likely to have had a prior history of angina, myocardial infarction (MI) and percutanous coronary intervention (PCI) [86.6 vs $37.7 \%$; 66.8 vs $21.8 \%$; 32.5 vs $10.5 \%$, respectively, $\mathrm{p}<0.0001]$. They were more likely to be type 2 diabetic, hypertensive and dyslipidemic [62.3 vs 37.6\%; 75.7 vs $47.8 \% ; 70.3$ vs $29.5 \%$, respectively, $\mathrm{p}<0.0001]$. Comor- bidities including previous stroke and peripheral arterial disease were more prevalent in the CABG group compared with the non-CABG group [ 9.5 vs $4.3 \%$; 9.5 vs $2.0 \%$ respectively, $\mathrm{p}<0.0001]$. CABG patients had also more chronic obstructive pulmonary disease (COPD) [ 8.9 vs $5.1 \%, \mathrm{p}=0.001]$ and were more likely to have been on dialysis $[2.0$ vs $0.9 \%$, $\mathrm{p}=0.046]$.

Table 2 summarizes the data on clinical presentation and investigations. ACS patients with prior CABG were more likely to present with advanced Killip class II-IV (33.1 vs $21.1 \%, P<0.0001)$ compared to ACS without prior CABG. Compared to the non-CABG patients, the CABG patients were more likely to present with UA and NSTEMI (46.6 vs $27.6 \% ; 41.4$ vs $31.6 \%$ respectively, $\mathrm{p}<0.0001)$. In addition, ACS patients with prior CABG were more likely to have left ventricular dysfunction (LVEF $\leq 40 \%$ : 49.4 vs $29.8 \%, P<$

Table 2. Baseline Clinical Presentations, Investigations and Discharge Diagnosis of Study Cohort

\begin{tabular}{|c|c|c|c|}
\hline & ACS without Prior CABG & ACS with Prior CABG & $\mathbf{P}$ \\
\hline \multicolumn{4}{|l|}{ Presentation (\%) } \\
\hline Ischemic type chest pain & 79.7 & 73.1 & 0.001 \\
\hline Atypical chest pain & 6.6 & 5.9 & 0.63 \\
\hline Dyspnea & 9.5 & 14.8 & 0.0005 \\
\hline Cardiac arrest & 2.4 & 2.8 & 0.53 \\
\hline \multicolumn{4}{|l|}{ BP Mean (SD) mmHg } \\
\hline Systolic & $138[120-160]$ & $137[120-160]$ & 0.55 \\
\hline Diastolic & $80[70-95]$ & $80[70-90]$ & $<0.0001$ \\
\hline Heart rate (mean) & $83[70-98]$ & $80[70$ - 97] & 0.20 \\
\hline Killip Classification (\%) & & & $<0.0001$ \\
\hline Killip I & 78.9 & 66.9 & \\
\hline Killip II-IV & 21.1 & 33.1 & \\
\hline \multicolumn{4}{|l|}{ Echocardiographic findings } \\
\hline Echocardiogram done (\%) & 61.9 & 51.5 & $<0.0001$ \\
\hline $\mathrm{LVEF} \leq 40 \%$ & 29.8 & 49.4 & $<0.0001$ \\
\hline \multicolumn{4}{|l|}{ Discharge Diagnosis (\%) } \\
\hline STEMI & 39.6 & 8.9 & $<0.0001$ \\
\hline NSTEMI & 31.6 & 41.4 & $<0.0001$ \\
\hline UA & 27.6 & 46.6 & $<0.0001$ \\
\hline LBBB MI & 1.2 & 3.0 & 0.002 \\
\hline \multicolumn{4}{|l|}{ Lab. Investigations } \\
\hline Total cholesterol (mg/dL) & $4.6[3.5-5.6]$ & $4.3[3.5-5.3]$ & $<0.0001$ \\
\hline $\mathrm{LDL}(\mathrm{mg} / \mathrm{dL})$ & $3.1[2.4-4.0]$ & $2.8[2.2-3.7]$ & $<0.0001$ \\
\hline $\mathrm{HDL}(\mathrm{mg} / \mathrm{dL})$ & $1.0[0.8-1.2]$ & $0.9[0.8-1.2]$ & 0.11 \\
\hline Triglycerides (mmol/L) & $1.6[1.1-2.3]$ & $1.5[1.1-2.3]$ & 0.54 \\
\hline Creatinine $(\mu \mathrm{mol} / \mathrm{L})$ & 88 [62-106] & $97[70-123]$ & $<0.0001$ \\
\hline
\end{tabular}

LVEF: left ventricular ejection fraction, STEMI: ST segment elevation myocardial infarction, NSTEMI: non ST segment elevation myocardial infarction, UA: unstable angina, LBBB MI: left bundle branch block myocardial infarction, LDL: Low-density lipoprotein cholesterol, HDL: High-density lipoprotein cholesterol. 
0.0001). On initial laboratory testing, the CABG patients tested with significantly lower levels of total and low-density lipoprotein (LDL) cholesterol and higher serum creatinine levels $(P<0.05)$.

Treatment patterns are presented in Table 3. For STEMI patients, approximately half of those with prior CABG received thrombolytic therapy $(48.7 \%)$ with no significant difference between the CABG and non-CABG groups $(P>$ $0.05)$. During the first $24 \mathrm{~h}$ following admission, CABG patients were more likely to be treated with angiotensin converting enzyme inhibitors/angiotensin receptor blockers (ACEI/ARB) (75.1 vs $67.3 \%, P<0.05)$; diuretics (45.5 vs $24.2 \%, P<0.0001)$ and nitrates $(89.3$ vs $80.8 \%, P<0.0001)$ than patients without prior $\mathrm{CABG}$, and slightly less likely to be treated with aspirin, beta-blockers and heparin. At the time of discharge, patients with prior CABG were less likely to be discharged on aspirin and beta-blockers, but more likely to be discharged on statins, nitrates and diuretics. Prior CABG patients compared with non-CABG patients under- went fewer coronary angiograms during the index hospitalization (15.9 vs $12.1 \%, P>0.05$ ) (Table 3 ).

ACS patients with CABG suffered more episodes of recurrent ischemia (Table 4) (13.9 vs 9.3\%, $P<0.05)$, heart failure (24.1 vs $15.7 \%, P<0.0001$ ), and requiring ventilator support ( 8.3 vs $4.6 \%, P<0.05)$. They received more inotropic support $(11.5$ vs $7.4 \%, P<0.001)$, had higher rates of major bleeds $(2.2$ vs $0.6 \%, P<0.05)$, strokes $(2.2$ vs $0.6 \%, P<0.0001)$ and in-hospital mortality rate $(5.6$ vs $3.5 \%, P<0.05)$ than the non-CABG patients.

In univariate analyses, patients with prior CABG were significantly more likely to have adverse events during their admission for ACS with significantly higher rates of recurrent ischemia, heart failure, requirement for mechanical ventilation and inotropic support, major bleeding, stroke and death (Table 4). After adjusting for differences in baseline characteristics and ACS type (Table 5), prior CABG was associated with about a 4-fold increased risk of recurrent

Table 3. Treatment Patterns for ACS Patients with and without Prior CABG

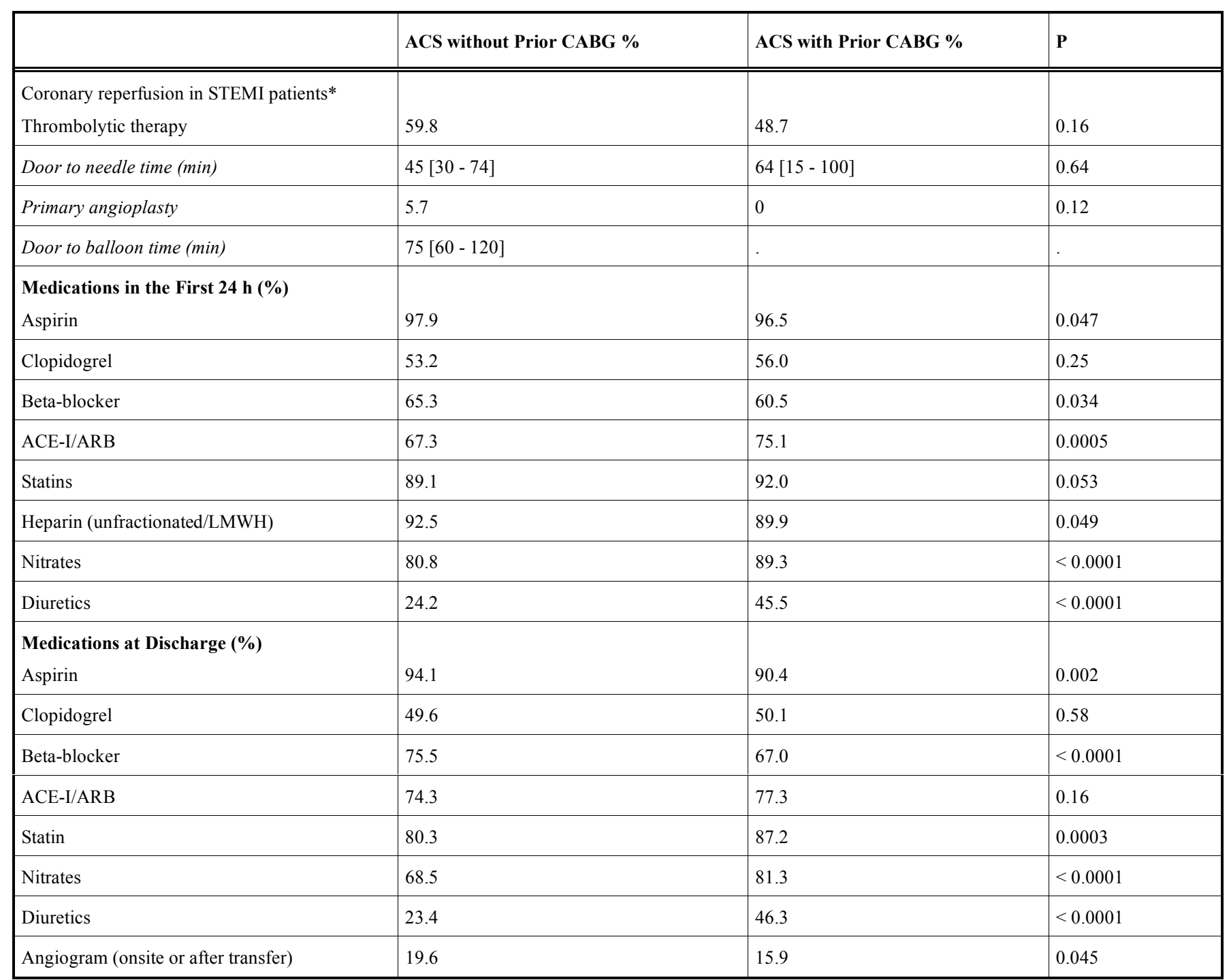

STEMI: ST segment elevation myocardial infarction, LMWH: low molecular weight heparin, ACE-I/ARB: angiotensin converting enzyme inhibitor/angiotensin receptor blocker. *Of 3,096 patients with STEMI, 41 had a history of prior CABG. 
Table 4. In-Hospital Course and outcomes for ACS Patients with and without Prior CABG

\begin{tabular}{|l|l|l|l|}
\hline & ACS without Prior CABG \% [n] & ACS with Prior CABG \% [n] & P \\
\hline \hline Recurrent ischemia & 9.3 & 13.9 & 0.001 \\
\hline Infarction/ Reinfarction & 2.5 & 2.2 & 0.68 \\
\hline Heart failure & 15.7 & 24.1 & $<0.0001$ \\
\hline Ventilation & 4.6 & 8.3 & 0.0005 \\
\hline IABP use & 0.7 & 0.7 & 0.99 \\
\hline Inotrope & 7.4 & 11.5 & 0.001 \\
\hline Cardiogenic shock & 5.0 & 7.0 & 0.059 \\
\hline Acute MR & 1.0 & 0.9 & 0.82 \\
\hline VSD/LV Rupture & 0.1 & 0.2 & 0.47 \\
\hline Major bleeding & 0.6 & 2.2 & 0.0002 \\
\hline Stroke & 0.6 & 2.2 & $<0.0001$ \\
\hline Death & 3.5 & 5.6 & 0.019 \\
\hline
\end{tabular}

IABP: intraaortic balloon pump, MR: mitral regurgitation, VSD/LV: ventricular septal defect/left ventricle.

Table 5. Adjusted in-Hospital outcomes in Patients with Acute Coronary Syndrome and Prior CABG Compared with those without Prior CABG

\begin{tabular}{|l|l|l|l|l|}
\hline In-Hospital Outcome & Odds Ratio & $\mathbf{9 5 \%}$ CI & P & Interaction P \\
\hline \hline Death & 1.55 & $0.95-2.54$ & 0.08 & 0.096 \\
\hline Recurrent ischemia & STEMI 4.08 & $2.27-7.33$ & 0.0004 & $<0.0001$ \\
& NSTE-ACS 0.94 & $0.66-1.33$ & $1.45-5.21$ & 0.009 \\
\hline Cardiogenic shock & STEMI 2.75 & $0.46-1.59$ & NS & 0.0001 \\
\hline Stroke & NSTE-ACS 0.85 & $1.19-6.21$ & 0.017 & 0.21 \\
\hline Major bleeding & 2.72 & $1.33-6.87$ & 0.008 & 0.17 \\
\hline
\end{tabular}

*The odds ratios are adjusted for age, gender, creatinine, ACS type (STEMI/LBBB MI vs NSTE-ACS) and all comorbidities, NS: non-significant.

ischemia and more than 2-fold increased risk of cardiogenic shock among patients with STEMI but not in patients NSTEACS ( $\mathrm{P}$ for interaction $<0.0001$ and 0.0087 , respectively). There was a trend for significant association between history of CABG and increased risk of death (OR 1.55, 95\%CI $0.95-$ 2.54, $\mathrm{P}=0.08)$.

\section{DISCUSSION}

Patient with prior CABG can develop acute coronary syndrome (ACS) [3, 11], with increasing numbers observed in recent years [5, 12-14]. Angiographic studies have demonstrated that vein graft occlusion and disease progression are temporally related to ACS. Between 10 and 15 years following surgery only $50-60 \%$ of vein grafts remain patent. Of these patent grafts, $45 \%$ showed angiographic evidence of atherosclerosis. Furthermore, $70 \%$ of these lesions reduce the graft lumen diameter by $50 \%$ or more [15-17].
The goal of the present analysis was to determine the clinical impact of ACS on a Middle Eastern cohort of patients with prior CABG. We found ACS patients with prior CABG were older and suffered from significantly more adverse comorbidities than the non-CABG ACS group. Stroke and PAD were more common, and the prior-CABG group had higher rates of angina, infarction and PCI suggesting that ACS CABG patients had a greater degree of coronary artery disease and generalized atherosclerosis. In addition, diabetes mellitus, hypertension and dyslipidemia were more prevalent in this cohort. Our clinical findings are consistent with and confirm those from other reports [6, 8, 13, 18]; though among Middle Eastern patients our study uncovered a higher frequency of hypertension and diabetes than previously observed elsewhere $[6-8,19]$.

Although ACS patients with prior CABG in the Gulf RACE presented more frequently with NSTEMI, heart fail- 
Table 6. Comparison of Gulf RACE with other Studies

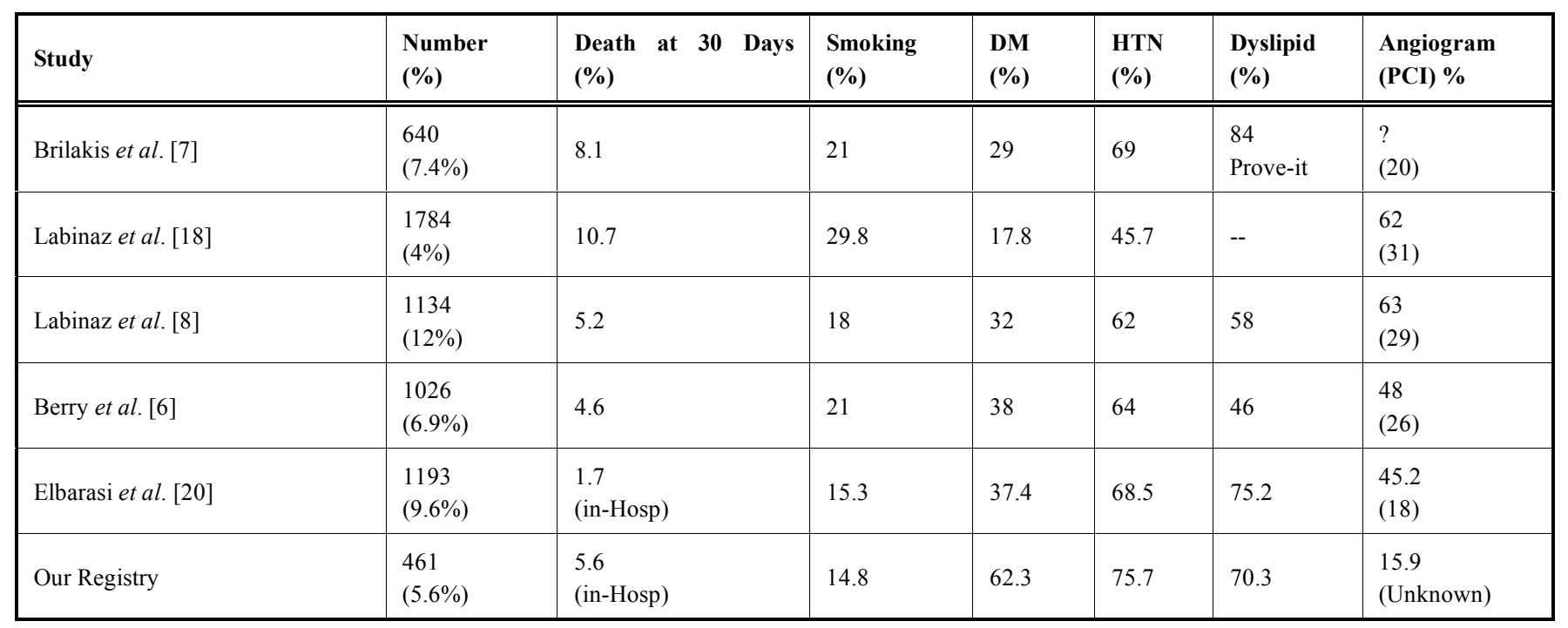

ure and ventricular dysfunction than those without prior $\mathrm{CABG}$, the rate of coronary catheterization during the index hospitalization was low compared to other studies [20].

Patients with prior CABG also had a more complicated in-hospital course with frequent episodes of recurrent ischemia, heart failure, major bleeding, and stroke. Thrombolysis was used more often than reported in other registries [6], and almost half the CABG patients and $60 \%$ of the non-CABG patients with STEMI had thrombolysis. In comparison, in the GRACE Registry [21] one-third of the patients with STEMI did not undergo reperfusion therapy. Reperfusion therapy was less likely to be used on angina-free patients, the elderly and those with diabetes, heart failure, or a previous MI or CABG. In our study, contrary to our expectations, the ACS patients with prior CABG underwent fewer catheterizations during the index hospitalization than those without prior CABG.

Our data showed that history of prior CABG surgery is independently associated with a higher risk of recurrent ischemia, shock, bleeding and stroke after adjusting for baseline variables. Moreover, prior $\mathrm{CABG}$ was associated with high risk of shock and recurrent ischemia in patients presenting with STEMI and not in non-ST elevation MI. This could suggests that the mechanism of acute $\mathrm{MI}$ in prior CABG patients maybe different and needs aggressive reperfusion and revascularization therapy. In a study by Grines et al., acute $\mathrm{MI}$ in patients with prior CABG was mainly due to thrombotic occlusion of a saphenous vein graft and conventional thrombolytic therapy may not be sufficient [22].

Compared with the 5 other studies (Table 6) [6-8, 18, $20]$, our Registry had the smallest percentage of coronary angiography and intervention during the index hospitalization of patients with ACS and prior CABG. We found a significantly different distribution of risk factors compared with Western data. In our study, diabetes and hypertension were the highest observed comorbid conditions among all 6 groups while smoking and dyslipidemia were in the midrange. The observed mortality in our group of 461 patients was in the mid-range of study results. Our in-hospital death rate was $5.6 \%$ while in the other studies it ranged from 1.7 to $10.7 \%$, nonetheless how the comorbidities affect mortality should still be examined.

ACS patients with prior CABG are a high-risk group and require special attention to decrease and prevent morbidity and mortality. They have different demographic characteristic, noteworthy risk factors, a significant past CAD history, and multiple comorbidities. We found that this patient group is evaluated less aggressively. Mortality rates are increased in this patient type probably due to higher prevalence of comorbidities, more generalized degree of atherosclerosis and less aggressive intervention.

Several limitations should be considered. First, angiographic assessment of all patients, an important factor in the decision-making for patients who are not amenable for any further revascularization due to the aggressiveness of disease, is not known. Second, neither the time of the CABG surgery nor the type of vessel used (arterial or venous graft) is known making it impossible to assess the graft patency rate. Thirdly, eventhough its known that age and several risk factor like diabetes, hypertension and dyslipidemia negatively affect the patency rate of CABG we don't have that data available. Despite these limitations, our findings were derived from a prospectively defined analysis of a large multi-centre registry and have important implications for management of ACS patients in real life.

\section{CONCLUSIONS}

Among the Gulf RACE cohort a history of prior CABG appears to have an important effect on prognosis in those who subsequently develop an ACS. Identifying this highrisk group will help improve treatment and prevention in the future.

\section{ACKNOWLEDGEMENTS}

Special thanks to Dr. Hisham Mahmoud (Medical Director - sanofi aventis, Gulf) for his support during the course of the study. 


\section{FUNDING}

Gulf RACE is a Gulf Heart Association project and was financially supported by sanofi aventis and Qatar Telecommunications Company. The sponsors had no role in study design, data collection or data analysis. The sponsors had no role in the writing of the report and submission of the manuscript.

\section{REFERENCES}

[1] Ferguson TB Jr, Hammill BG, Peterson ED, DeLong ER, Grover FL. A decade of change--risk profiles and outcomes for isolated coronary artery bypass grafting procedures, 1990-1999: a report from the STS National Database Committee and the Duke Clinical Research Institute. Society of Thoracic Surgeons. Ann Thorac Surg 2002; 73: 480-9.

[2] Long-term results of prospective randomised study of coronary artery bypass surgery in stable angina pectoris. European Coronary Surgery Study Group. Lancet 1982; 2: 1173-80.

[3] Varnauskas E. Twelve-year follow-up of survival in the randomized European Coronary Surgery Study. N Engl J Med 1988; 319: 332-7.

[4] The Society for Cardiothoracic Surgery in Great Britain and Ireland. Sixth National Adult Cardiac Surgical Database Report: Demonstrating Quality. 2008. http: //www.scts.org/documents/ PDF/Sixth NACSD report 2008 with c.pdf (Accessed 30/10/2010).

[5] Rogers WJ, Canto JG, Lambrew CT, et al. Temporal trends in the treatment of over 1.5 million patients with myocardial infarction in the US from 1990 through 1999: the National Registry of Myocardial Infarction 1, 2 and 3. J Am Coll Cardiol 2000; 36: 2056-63.

[6] Berry C, Pieper KS, White HD, et al. Patients with prior coronary artery bypass grafting have a poor outcome after myocardial infarction: an analysis of the VALsartan in acute myocardial iNfarcTion trial (VALIANT). Eur Heart J 2009; 30: 1450-6.

[7] Brilakis ES, de Lemos JA, Cannon CP, et al. Outcomes of patients with acute coronary syndrome and previous coronary artery bypass grafting (from the Pravastatin or Atorvastatin Evaluation and Infection Therapy [PROVE IT-TIMI 22] and the Aggrastat to Zocor [A to Z] trials). Am J Cardiol 2008; 102: 552-8.

[8] Labinaz M, Kilaru R, Pieper K, et al. Outcomes of patients with acute coronary syndromes and prior coronary artery bypass grafting: results from the platelet glycoprotein IIb/IIIa in unstable angina: receptor suppression using integrilin therapy (PURSUIT) trial. Circulation 2002; 105: 322-7.

[9] Zubaid M, Rashed WA, Almahmeed W, et al. Management and outcomes of Middle Eastern patients admitted with acute coronary syndromes in the Gulf Registry of Acute Coronary Events (Gulf RACE). Acta Cardiol 2009; 64: 439-46.
[10] Zubaid M, Rashed WA, Al-Khaja N, et al. Clinical presentation and outcomes of acute coronary syndromes in the gulf registry of acute coronary events (Gulf RACE). Saudi Med J 2008; 29: 251-5.

[11] Sabik JF, 3rd, Blackstone EH, Gillinov AM, Smedira NG, Lytle $\mathrm{BW}$. Occurrence and risk factors for reintervention after coronary artery bypass grafting. Circulation 2006; 114: I454-60.

[12] Babaev A, Frederick PD, Pasta DJ, Every N, Sichrovsky T, Hochman JS. Trends in management and outcomes of patients with acute myocardial infarction complicated by cardiogenic shock. JAMA 2005; 294: 448-54.

[13] Goldberg RJ, Currie K, White K, et al. Six-month outcomes in a multinational registry of patients hospitalized with an acute coronary syndrome (the Global Registry of Acute Coronary Events [GRACE]). Am J Cardiol 2004; 93: 288-93.

[14] Granger CB, Goldberg RJ, Dabbous O, et al. Predictors of hospital mortality in the global registry of acute coronary events. Arch Intern Med 2003; 163: 2345-53.

[15] Bourassa MG, Enjalbert M, Campeau L, Lesperance J. Progression of atherosclerosis in coronary arteries and bypass grafts: ten years later. Am J Cardiol 1984; 53: 102C-7C.

[16] Fitzgibbon GM, Kafka HP, Leach AJ, Keon WJ, Hooper GD, Burton JR. Coronary bypass graft fate and patient outcome: angiographic follow-up of 5,065 grafts related to survival and reoperation in 1,388 patients during 25 years. J Am Coll Cardiol 1996; 28: 616-26.

[17] Goldman S, Zadina K, Moritz T, et al. Long-term patency of saphenous vein and left internal mammary artery grafts after coronary artery bypass surgery: results from a Department of Veterans Affairs Cooperative Study. J Am Coll Cardiol 2004; 44: 2149-56.

[18] Labinaz M, Sketch MH Jr, Ellis SG, et al. Outcome of acute STsegment elevation myocardial infarction in patients with prior coronary artery bypass surgery receiving thrombolytic therapy. Am Heart J 2001; 141: 469-77.

[19] Franklin K, Goldberg RJ, Spencer F, et al. Implications of diabetes in patients with acute coronary syndromes. The Global Registry of Acute Coronary Events. Arch Intern Med 2004; 164: 1457-63.

[20] Elbarasi E, Goodman SG, Yan RT, et al. Management patterns of non-ST segment elevation acute coronary syndromes in relation to prior coronary revascularization. Am Heart J 2010; 159: 40-6.

[21] Eagle KA, Goodman SG, Avezum A, Budaj A, Sullivan CM, Lopez-Sendon J. Practice variation and missed opportunities for reperfusion in ST-segment-elevation myocardial infarction: findings from the Global Registry of Acute Coronary Events (GRACE). Lancet 2002; 359: 373-7.

[22] Grines CL, Booth DC, Nissen SE, et al. Mechanism of acute myocardial infarction in patients with prior coronary artery bypass grafting and therapeutic implications. Am J Cardiol 1990; 65: $1292-6$. 